Pacific Journal of Mathematic 


\section{ON THE MEASURE OF NORMAL FORMULAS}

\section{ROBERT MCNAUghtoN}

1. Introduction. Quine has recently found (in [1], [2] and [3]) a reasonably practical method which yields the simplest normal equivalent of a given truth functional formula. The problem of this paper is to find a practical method which yields the simplest normal formula with a given measure. Roughly, the measure of a formula is the number of $T^{\prime} \mathrm{s}$ in the column under the formula in a truth table which has $2^{a}$ rows; these rows represent all possible assignments of $T^{\prime}$ s and $F^{\prime} \mathrm{s}$ to $d$ letters including all the letters of the formula and perhaps others. The problem, which is rather difficult, arises in the design of certain networks in digital computers (described at the end of $\S 2$ ) as part of a more general problem which is all the more difficult. Networks, however, are not discussed at all in the remainder of the paper, where the main problem is attacked as a problem in pure logic. I have had no success in obtaining a method which is generally satisfactory, but have succeeded in proving a few theorems which will probably be indispensable in any future attack on the problem.

2. The problem and its origin. Most of the terminology which I shall use is Quine's. Where it conflicts with Quine's terminology of [1], [2] and [3] I shall explicitly say so; on the other hand, I shall not presuppose that the reader is familiar with any of these papers. An italicized word appearing in a sentence of this paper is defined in that sentence. In this section a sentence without an italicized word is often a theorem which is either well known or obvious.

A formula is made up in the usual manner from the letters $A_{1}, \cdots$, $A_{a}$ by means of negation, conjunction and disjunction (or alternation). For any formulas $\Phi_{1}, \cdots, \Phi_{n}, n \geqq 2, \Phi_{1}$ is the negation of $\Phi_{1}, \Phi_{1} \Phi_{2} \cdots \Phi_{n}$ is the conjunction of $\Phi_{1}, \cdots, \Phi_{n}$ (these being conjuncts), and $\Phi_{1} \vee \Phi_{2} \vee \cdots$ $\vee \Phi_{n}$ is the disjunction (called 'alternation' by Quine) of $\Phi_{1}, \cdots, \Phi_{n}$ (these being disjuncts). (I assume that the reader is familiar enough with the general literature to see how the circularity of definition in the last two sentences can be avoided.) A letter or its negation is a literal. If a formula is a disjunction, then the disjuncts are clauses; if it is not a disjunction, the formula itself is its only clause. A formula all of whose clauses are literals or conjunctions of literals is a normal formula. (For Quine a clause of a normal formula cannot have

Received January 30, 1956. This paper is an abridged version of report No. 1, contract DA-04-200-ORD-436 (Applied Mathematics and Statistics Laboratory, Stanford, California), sponsored by the Office of Ordnance Research. 
repetitious letters.)

A formula is consistent if it comes out true under some interpretation of its letters. An inconsistent normal formula, then, must be one in which every clause is inconsistent; a clause of a normal formula is inconsistent if and only if there is a letter appearing both with and without a bar. An inconsistent clause can be omitted from any consistent normal formula and the resulting formula is equivalent to the original. A clause of a normal formula subsumes another if every literal of the second is a literal of the first. Any clause which subsumes another in a normal formula can be omitted and the resulting formula is equivalent to the original. A literal which has occurred previously in the same clause can be omitted and the resulting clause is equivalent to the original clause; hence, the resulting formula is equivalent to the original formula. A normal formula in which no clause subsumes another, no clause is inconsistent, and no clause contains a repeated literal, is an apparently irredundant normal formula. An irredundant normal formula is one in which no literal or clause can be omitted without sacrificing equivalence. Some apparently irredundant formulas are not irredundant as example 1 or example 2 of [1] is enough to show. An interclausally consistent formula is one in which the conjunction of any two clauses is consistent. A normal formula is interclausally consistent if and only if no letter appears in it at least once with a bar and at least once without a bar.

A normal formula is developed with respect to the letters $A_{1}, \cdots$, $A_{a}$ if every clause has one and only one occurrence of each of these letter. Every consistent formula $\Phi$ containing no letters other than $A_{1}$, $\cdots, A_{d}$ can be transformed into an irredundant normal formula which is developed with respect to the letters $A_{1}, \cdots, A_{d}$; the number of clauses in the letter is the measure of $\Phi$, or $m(\Phi)$. If $\Phi$ is inconsistent, then the measure of $\Phi$ is 0 . In general $m(\Phi)$ depends on $d$, but there is no need to make this dependence explicit in the notation in most of this paper. Where the notation $m(\Phi)$ is used, it is assumed that $\Phi$ contains only (perhaps not all) the letters $A_{1}, \cdots, A_{d}$. If a truth table is constructed for $\Phi$ with $2^{a}$ rows, representing the $2^{d}$ assignments of truth values to $A_{1}, \cdots, A_{d}$, then there will be $m(\Phi) T^{\prime}$ s in the column for $\Phi$.

Two formulas $\Phi$ and $\Psi$ are isomorphic if there is a one-to-one mapping $f$ of the set of literals of $\Phi$ onto the set of literals of $\psi$ such that, if both $A_{i}$ and $\overline{A_{i}}$ occur in $\Phi$ and if $f\left(A_{i}\right)=A_{j}$ then $f\left(\overline{A_{i}}\right)=\overline{A_{j}}$, and if $f\left(A_{i}\right)=\overline{A_{j}}$ then $f\left(\overline{A_{i}}\right)=A_{j}$; and such that a formula $\Psi^{\prime}$ can be obtained from $\Phi$ by replacing each literal by its image under $f$, and $\Psi$ can be obtained from $\Psi^{\prime}$ by changing the order of conjunts of zero or more conjunctions and changing the order of disjuncts of zero or more disjunctions, Thus $A_{1} A_{2} \bigvee \overline{A_{1}} A_{3} A_{4} \vee \overline{A_{3}} A_{4} A_{5}$ is isomorphic to $A_{1} A_{5} \overline{A_{6}} \vee A_{4} \overline{A_{5}} \overline{A_{6}}$ 
$\bigvee A_{2} \overline{A_{1}}$; here $f$ is the mapping such that $f\left(A_{1}\right)=\overline{A_{1}}, f\left(A_{2}\right)=A_{2}, f\left(A_{3}\right)=A_{5}$, $f\left(A_{4}\right)=\bar{A}_{6}, f\left(A_{5}\right)=A_{4}$. If no letters except $A_{1}, \cdots, A_{d}$ occur in $\Phi$ and $T$, and if $\Phi$ and $\Psi$ are isomorphic, then $m(\Phi)=m(\Psi)$. (If this fact is not obvious enough to the reader, it is proved for the case in which $\Phi$ and $\Psi$ are normal formulas as Theorem 2.4.)

For the purposes of this paper the word " simplicity" need not be, and is not, defined precisely. Let us understand merely that simplicity of a normal formula depends on the number of clauses and the number of literals in each clause.

A practical solution to the problem of finding the simplest normal formula with a given measure would have some application to the design of certain parts of digital computers. Dr. Montgomery Phister, Jr. of the Ramo Wooldridge Corporation has suggested the following problem which was the initial stimulus for the research for this paper.

Suppose that one is to devise a circuit with $n$ outputs in such a way that in each of $m$ given time intervals each output is to be in state 1 . or state 0 as specified. The circuit engineer can select his inputs in any way he chooses, so that each input is either 0 or 1 in each interval. But he must do so in such a way that each output is a function of the inputs and the circuit is the most economical. If certain kinds of diode circuit are used, then the part of the circuit which relates any output to the inputs must be constructed as a normal formula.

The problem of finding the simplest normal formula with a given measure is relevant to this problem, even though a practical solution to the former would not necessarily mean a practical solution to the latter. If the number of intervals is between $2^{a-1}+1$ and $2^{a}$ inclusive and if each time interval itself is to be a unique function of the inputs, then there must be $d$ inputs. With these assumptions, a practical way of choosing inputs so as to minimize the circuit for just one output is easily obtainable if there is a practical way of finding the simplest normal formula with a given measure. For example, if there are 16 time intervals and there is to be one output in state 1 in exactly 5 intervals, then it is necessary to find, for $d=4$, a simplest formula whose measure is 5 ; in this case, $A_{1} A_{2} \vee A_{1} A_{3} A_{4}$ seems to be a formula.

3. Calculation of the measure of a formula. There is a straightforward way of calculating the measure of a normal formula which is somewhat simpler than actually expanding it into a developed normal formula. At the basis of this method is an easily proved theorem relating the measures of two formulas, their conjunction and their disjunction.

THEOREM 3.1. For any formulas $\Phi$ and $\Psi$ 


$$
m(\Phi \bigvee \psi)=m(\Phi)+m(\Psi)--m(\Phi \Psi)
$$

Proof. Consider the developed normal formulas with respect to $d$ variables equivalent to the four formulas concerned, $\Phi^{*}, \Psi^{*},(\Phi \bigvee \Psi)^{*}$, $(\Phi \Psi)^{*}$. The number of clauses in $(\Phi \bigvee \Psi)^{*}$ can be counted by counting the number of clauses in $\Phi^{*}$ (which is assumed to be 0 if $\Phi$ is inconsistent) and then counting the number of clauses in $\Psi^{*}$, remembering that any clauses which these have in common have been counted twice. But the number of clauses which $\Phi^{*}$ and $\Psi^{*}$ have in common is precisely the number of clauses in $(\Phi \Psi)^{*}$, or 0 if $\Phi \Psi$ is inconsistent, which in either case is $m(\Phi \Psi)$. Hence Theorem 3.1 follows.

THEOREM 3.2. If $\Phi$ is a conjunction of $j$ distinct literals, no two of which are of the same letter, then $m(\Phi)=2^{a-j}$.

This theorem follows readily from well known properties of truth tables or developed normal formulas.

For the remainder of this paper let $\Phi$ be a normal formula. Let $\varphi_{1}, \cdots, \varphi_{k}$ be the clauses of $\Phi$ in the order of their appearance in $\Phi$. Let $\Phi_{x}, 1 \leqq x \leqq k$, be the normal formula $\varphi_{1} \vee \varphi_{2} \vee \cdots \vee \varphi_{x}$. Thus $\Phi_{k}$ is $\Phi$. Let $C(\Phi)$ be the set of all clauses of $\Phi$. For any $S \leqq C(\Phi)$ let $j_{S}$ be the total number of distinct letters appearing in the clauses of $S$. Let $i_{S}$ be 0 if at least one letter appears in at least one clause with a bar and in at least one clause without a bar, that is, if the conjunction of all the clauses of $S$ is inconsistent. If there is no such letter, let $i_{S}$ be 1 if there are an odd number of clauses in $S$ or -1 if there are an even number of clauses in $S$.

\section{THEOREM 3.3 .}

$$
m(\Phi)=\sum_{S \leqq C(\Phi)} i_{s} 2^{a-j_{S}},
$$

the summation being taken only over nonempty subsets $S$.

The proof is by induction on the number $k$ of clauses in $\Phi$. If $k$ $=1$, then there is only one $S$ to be considered, namely, the unit set of the one clause. If there is a letter which appears both with and without a bar, $i_{S}$ is 0 and so is $m(\Phi)$. If there is no such letter, then $m(\Phi)=2^{a-j_{S}}$, by Theorem 3.2 .

Suppose that $k>1$ and suppose that Theorem 3.3 holds for all formulas having fewer than $k$ clauses. By Theorem 3.1,

$$
m(\Phi)=m\left(\Phi_{k-1}\right)+m\left(\varphi_{k}\right)-\left(\Phi_{k-1} \varphi_{k}\right) .
$$


Now $\Phi_{k-1} \varphi_{k}$ is not a normal formula unless $k-1=1$. If $k-1>1$ let $\Psi$ be the normal formula which is equivalent to $\Phi_{k-1} \varphi_{k}$ obtained by distributing the conjunct $\varphi_{k}$ over the clauses of $\Phi_{k-1}$, and if $k-1=1$, let $\Psi$ be $\Phi_{k-1} \varphi_{k}$. Thus $\Psi$ will have $k-1$ clauses and, for each $h \leqq k-1$, the $h^{\text {th }}$ clause of $\Psi$ will have the literals of the $h^{\text {th }}$ clause of $\Phi_{k-1}$ and those of $\varphi_{k}$, but no others. Now since $\Psi$ is equivalent to $\Phi_{k-1} \varphi_{k}$,

$$
m(\Psi)=m\left(\Phi_{k-1} \varphi_{k}\right) .
$$

By the inductive hypothesis and Theorem 3.2,

$$
\begin{aligned}
& m\left(\Phi_{k-1}\right)=\sum_{S \subseteq C\left(\Phi_{k-1}\right)} i_{S} 2^{d-j_{S}} \\
& m\left(\varphi_{k}\right)=i_{C\left(\varphi_{k}\right)} 2^{d-j_{C\left(\varphi_{k}\right)}},
\end{aligned}
$$

and

$$
m(\Psi)=\sum_{S \subseteq C(\Psi)} i_{S} 2^{d-j},
$$

where in each case only nonempty subsets $S$ are considered.

Making substitutions in (1), justified by (2), (3), (4) and (5), we get

$$
m(\Phi)=\sum_{S \subseteq C\left(\Phi_{k-1}\right)} i_{S^{2}} 2^{d-j_{S}}+i_{C\left(\varphi_{k}\right)} 2^{a-j_{C\left(\varphi_{k}\right)}}-\sum_{S \subseteq \sigma(\varphi)} i_{S} 2^{a-j_{S}} .
$$

It remains only to show that we can equate the expression $\sum_{S \subseteq C(\Phi)} i_{S} 2^{a-j} S$ with the right side of (6). But these expressions are equal, term by term, as can be seen as follows. For every $S$ in $C(\Phi)$, either $S$ does not contain $\varphi_{k}$ (case I), $S$ contains $\varphi_{k}$ and other clauses (case II), or $S$ contains $\varphi_{k}$ only (case III). In case III, the summand $i_{S} 2^{a-j}$ is $_{S}$ the middle term of the right side of (6). In case I, $S \in C\left(\Phi_{k-1}\right)$ and so the summand $i_{S} 2^{a-j_{S}}$ occurs as a summand in the first term on the right side of (6). In case II, finally, suppose $S$ contains besides $\varphi_{k}$ the $g_{1}^{\text {th }}$, $\cdots, g_{n}^{\text {th }}$ clauses of $\Phi$. Then consider $S^{\prime}$, the set containing the $g_{1}^{\text {th }}, \cdots$, $g_{n}^{\text {th }}$ clause of $\Psi$. The literals appearing in these clauses are exactly the literals appearing in the clauses of $S$. A letter will appear both with a bar and without a bar in $S$ if and only if it does in $S^{\prime}$. Hence, $i_{S^{\prime}}=0$ if and only if $i_{S}=0$. There an odd (even) number of clauses in $S$ if and only if there are an even (odd) number in $S^{\prime}$ since $S$ has just one more clause than $S^{\prime}$. Hence the summand $i_{s} 2^{d-j}$ occurs negatively as a summand in the third term of the right side of (6). It is easy to see that this correspondence is one to one and that the equality of the two expressions is established.

THEOREM 3.4. If $\Phi$ and $\Psi$ are normal formulas, and if $\Phi$ is iso- 
morphic to $\Psi$, then $m(\Phi)=m(\Psi)$.

Proof. The corresponding clause in $\Psi$ to a clause $\varphi$ in $\Phi$ is the clause which contains those literals into which the literals of $\varphi$ are mapped. Consider any set $S$ of clauses of $\Phi$ and the corresponding set of clauses $S^{\prime}$ in $\Psi$. The total number of distinct letters of $S$ equals the total number of distinct letters of $S^{\prime}$. There is a letter appearing with a bar and without a bar in $S$ if and only if there is such a letter appearing in $S^{\prime}$. And, of course, the number of clauses of $S$ is the same as the number of clauses of $S^{\prime}$. Thus the expressions for $m(\Phi)$ and $m(\Psi)$ as given by Theorem 3.3 will be the same, term by term.

The formula $\Psi$ implies $\Gamma$ if every assignment of truth values which makes $\Psi$ true also makes $\Gamma$ true. As is well known, $\Psi$ implies $\Gamma$ if and only if every clause of a developed normal formula equivalent to $\Psi$ is a clause of a developed normal formula with respect to the same variables equivalent to $\Gamma$. The formula $\Psi$ is equivalent to $\Gamma$ is $\Psi$ implies $\Gamma$ and $\Gamma$ implies $\Psi$. The formulas $\Psi$ and $\Gamma$ are equivalent if and only if they are equivalent to a common developed normal formula. Theorems 3.5, 3.6 and 3.7 are direct consequences of these remarks.

THeOREM 3.5. For any formulas $\Psi$ and $\Gamma, m(\Psi \vee \Gamma) \geq m(\Psi)$. The equality holds when, and only when, $\Gamma$ implies $\Psi$.

TheoRem 3.6. $m(\Psi \Gamma) \leqq m(\Psi)$. The equality holds when, and only when, $\Psi$ implies $\Gamma$.

THEOREM 3.7. If $\Gamma$ implies $\Psi$, then $m(\Gamma) \leqq m(\Psi)$. The equality holds when, and only when, $\Gamma$ and $\Psi$ are equivalent.

4. Bounds on the measure of a formula with a given structure. If $j_{1} \leqq j_{2} \leqq \cdots \leqq j_{k}$, then a formula has the structure $\left\langle j_{1}, j_{2}, \cdots, j_{k}\right\rangle$ if and only if it is an apparently irredundant normal formula with $k$ clauses which have, in the order in which they appear in the formula, $j_{1}, \cdots, j_{k}$ literals. Note that a formula has some structure if and only if it is normal, it is apparently irredundant, and its clauses are in order of nondecreasing length. In finding a simplest normal formula we need only consider formulas which have some structure; for every normal formula which does not is equivalent to, and is no simpler than, a normal formula which does. In this section I shall give an upper bound and a lower bound on the measure of formula with a given structure. This result will be convenient in some cases where one is trying to determine a simplest formula with a given measure.

TheOREM 4.1. If $\Phi$ has the structure $\left\langle j_{1}, \cdots, j_{k}\right\rangle$, then 


$$
m(\Phi) \leqq 2^{d-j_{1}}+\cdots+2^{a-j_{k}} .
$$

The proof is by induction on $k$. For $k=1$, if $\Phi$ is of structure $\left\langle j_{1}\right\rangle$, then since $\Phi$ is apparently irredundant, $m(\Phi)=2^{a-j_{1}}$ by Theorem 2.2. I shall assume that Theorem 4.1 is true about all structures whose formulas have less than $k$ clauses, and show that it is true about the structure $\left\langle j_{1}, \cdots, j_{k}\right\rangle$. If $\Phi$ has the structure $\left\langle j_{1}, \cdots, j_{k}\right\rangle$, then, by inductive hypothesis, $m\left(\Phi_{k-1}\right) \leqq 2^{d-j_{1}}+\cdots+2^{d-j_{k-1}}$, and $m\left(\varphi_{k}\right)=2^{d-j_{k}}$. But, by Theorem 3.1,

$$
m(\Phi) \leqq m\left(\Phi_{k-1}\right)+m\left(\varphi_{k}\right) \leqq 2^{a-j_{1}}+\cdots+2^{a-j_{k-1}}+2^{a-j_{k}} .
$$

(Note that, for any formula $\Phi$ with structure $\left\langle j_{1}, \cdots, j_{k}\right\rangle, m(\Phi)=2^{a-j_{1}}$ $+\cdots+2^{d-j_{k}}$ if and only if the conjunction of every pair of clauses is inconsistent. It can be proved that such a formula exists if and only if $2^{a-j_{1}}+\cdots+2^{a-j_{k}} \leqq 2^{a}$.)

Theorem 4.2, 4.3 and 4.4 are, in effect, lemmas to Theorem 4.5 which establishes a lower bound on formulas with a given structure.

THEOREM 4.2. If $\Phi$ is not interclausally consistent, if the number of distinct literals of $\Phi$ does not exceed $d$, and if $\Phi$ has some structure, then there is an interclausally consistent normal formula with the same structure as $\Phi$ but with no greater measure.

Proof. If $\Phi$ is not interclausally consistent, then there is at least one letter in $\Phi$ appearing both with and without a bar I shall prove Theorem 4.2 by proving that there is a formula $\Phi^{\prime}$ with the same structure as $\Phi$ with exactly one less letter appearing both with and without a bar, such that $m\left(\Phi^{\prime}\right) \leqq m(\Phi)$. Suppose $A_{n}$ appears both with and without a bar in $\Phi$. Let $\Phi^{\prime}$ be $\Phi$ with every occurrence of $\overline{A_{n}}$ replaced by a variable $A_{p}$ with does not appear in $\Phi$. Since $\Phi$ has some structure, it is apparently irredundant, and so $A_{n}$ never appears both with and without a bar in any one clause of $\Phi$. Hence $\Phi$ is equivalent to $A_{n} \psi \vee \bar{A}_{n} \Gamma \vee \Omega$ and $\Phi^{\prime}$ is equivalent to $A_{n} \psi \vee A_{p} \Gamma \vee \Omega$, where $\psi, \Gamma$, and $\Omega$ are normal formulas in which $A_{n}, \bar{A}_{n}$, and $A_{p}$ do not occur. By Theorem 2.1, then, the following hold.

$$
\begin{aligned}
& m(\Phi)=m\left(\bar{A}_{n} \Gamma\right)+m\left(A_{n} \psi \bigvee \Omega\right)-m\left(\bar{A}_{n} \Gamma\left(A_{n} \psi \vee \Omega\right)\right) \\
& m\left(\Phi^{\prime}\right)=m\left(A_{p} \Gamma\right)+m\left(A_{n} \psi \vee \Omega\right)-m\left(A_{p} \Gamma\left(A_{n} \psi \bigvee \Omega\right)\right)
\end{aligned}
$$

We have, $m\left(A_{p} \Gamma\right)=m\left(\overline{A_{n}} \Gamma\right)$ since $A_{p} \Gamma$ and $\overline{A_{n}} \Gamma$ are isomorphic and can easily be converted into isomorphic normal formulas. Therefore we can concentrate on the last term of each equation. $\bar{A}_{n} \Gamma\left(A_{n} \psi \vee \Omega\right)$ is equivalent to $\bar{A}_{n} \Gamma \Omega$, and $A_{p} \Gamma\left(A_{n} \psi \vee \Omega\right)$ is equivalent to $A_{n} A_{p} \Gamma \psi \vee A_{p} \Gamma \Omega$. 
Also $m\left(\overline{A_{n}} \Gamma \Omega\right)=m\left(A_{p} \Gamma \Omega\right)$, the formulas being isomorphic. Finally $m\left(A_{p} \Gamma \Omega\right) \leqq m\left(A_{n} A_{p} \Gamma \psi \bigvee A_{p} \Gamma \Omega\right)$, by Theorem 3.5. Thus $m\left(\Phi^{\prime}\right) \leqq m(\Phi)$, $\Phi^{\prime}$ has the same structure as $\Phi$, and the number of literals of $\Phi^{\prime}$ equals the number of literals of $\Phi$.

Now if $\Phi^{\prime}$ still has at least one letter with and without a bar, I construct $\Phi^{\prime \prime}$, related to $\Phi^{\prime}$ as $\Phi^{\prime}$ is to $\Phi$, and so forth. Eventually I shall obtain a formula $\Phi^{(q)}$ which has no letters appearing both with and without a bar, has the same structure as $\Phi$ and has demonstrably no greater measure. It is obvious enough that the number of variables in $\Phi^{(q)}$ will not exceed $d$ if the hypothesis of the theorem is satisfied.

(Two things can be noted. First the formula $\Phi^{(q)}$ can easily be constructed from $\Phi$ as follows: supposing (without loss of generality) that $A_{1}, \cdots, A_{q}$ are the variables which appear both with and without bars and $A_{q+1}, \cdots, A_{r}$ are the other variables of $\Phi$, replace $\overline{A_{1}}, \cdots, \bar{A}_{t}$ by $A_{r+1}, \cdots, A_{r+q}$ respectively. Second, if we prefer, we can delete all the bars from $\Phi^{(q)}$ and the resulting formula will have the same measure, being isomorphic to $\Phi^{(q)}$. In summary, then, given a formula $\Phi$ which satisfies the hypothesis of Theorem 4.2 it is an easy matter to write down another formula without bars, with the same structure and with the same structure and with no greater measure.)

Theorem 4.3. If $\Psi, \Gamma$ have no letters in common, then

$$
m(\Psi \Gamma)=\frac{m(\Psi) \cdot m(\Gamma)}{2^{a}},
$$

Proof. Suppose (without loss of generality) that $A_{1}, \cdots, A_{n}$ are the letters occurring in $\Psi$; then every letter appearing in $\Gamma$ is one of the letters $A_{n+1}, \cdots, A_{a}$. From well known logical laws, the developed normal equivalent $\Psi^{\prime}$ of $\Psi$ with respect only to the letters $A_{1}, \cdots, A_{n}$ has $m(\Psi) / 2^{a-n}$ clauses. And the developed normal equivalent $\Gamma^{\prime}$ of $\Gamma$ with respect to the letters $A_{n+1}, \cdots, A_{d}$ has $m(\Gamma) / 2^{n}$ clauses. $\Psi \Gamma$ is equivalent to $\Psi^{\prime} \Gamma^{\prime}$; the developed normal equivalent of these can be obtained from the latter by the distributive law for disjunction over conjunction; the number of clauses will be the product of the number of clauses of $\Psi^{\prime}$ and $\Gamma^{\prime}$, which is $m(\Psi) m(\Gamma) / 2^{a}$. Since this last formula is the developed normal equivalent of $\Psi \Gamma$ with respect to $A_{1}, \cdots, A_{d}$, this number is the measure of $\Psi \Gamma$. (For example, if $d=5, \Psi$ is $A_{1} \vee A_{2}$ and $\Gamma$ is $A_{1} A_{5}$, then $n=2, \Psi^{\prime}$ is $A_{1} A_{2} \bigvee A_{1} \overline{A_{2}} \bigvee \overline{A_{1}} A_{2}$ and $\Gamma^{\prime}$ is $A_{3} A_{4} A_{5} \bigvee \overline{A_{3}} A_{4} A_{5}$. The result of " multiplying out" $\Psi^{\prime}$ and $\Gamma^{\prime}$ yields the developed normal equivalent with respect to $A_{1}, \cdots, A_{5}$ of $\Psi \Gamma$.)

Theorem 4.4. If $k<d$, the formula $A_{1} \vee A_{2} \vee \cdots \vee A_{k}$ has the 
maximum measure $2^{a-1}+2^{a-2}+\cdots+2^{a-k}$ of all interclausally consistent normal formulas having exactly $k<d$ clauses; the formula $A_{1} \bigvee A_{2} \vee \cdots$ $\vee A_{d}$ has the maximum measure $2^{a-1}+2^{a-2}+\cdots+2^{0}$ of all interclausally consistent normal formulas having $d$ or more clauses.

Proof. The formula mentioned in the second part of the theorem is equivalent to $-\left(\bar{A}_{1} \bar{A}_{2} \cdots \bar{A}_{d}\right)$. Therefore its developed normal form has all the $2_{a}$ clauses except one, and therefore its measure is $2^{a}-1$. This measure is a maximum for all interclausally consistent formulas since the one higer measure, $2^{a}$, is that of a tautology, which is never interclausally consistent.

The formula of the first part of the theorem, when developed with respect to $A_{1}, \cdots, A_{k}$, has $2^{k}-1$ clauses (by the first two sentences of the above paragraph with ' $k$ ' for ' $d$ '). From this we obtain an equivalent formula developed with respect to all $d$ letters by developing each clause into $2^{a-k}$ clauses. The measure of the formula, therefore, is

$$
2^{d-k}\left(2^{k}-1\right)=2^{d}-2^{a-k}=2^{a-1}+2^{d-2}+\cdots+2^{a-k} .
$$

Now every interclausally consistent formula with $k$ clauses not isomorphic to $A_{1} \vee \cdots \vee A_{k}$ either has at least one clause with more than one literal or has one literal in every clause with some repetitions of clauses. In the latter case, the formula is equivalent to $A_{1} \vee \cdots \vee A_{j}$, for some $j<k$, whose measure is $2^{a}+2^{a-1}+\cdots+2^{a-j}<2^{a}+\cdots+2^{a-k}$. I dispose of the former case by showing that, in an interclausally consistent formula, if every clause containing more than one literal is replaced by just one of its literals then the measure of the formula is not decreased. Suppose the formula $\Psi \vee A_{i_{1}} \varphi_{i_{1}} \vee \cdots \vee A_{i_{n}} \varphi_{i_{n}}$ is thus replaced by $\Psi \vee A_{i_{1}} \vee \cdots \vee A_{i_{n}}$. The latter is implied by the former and hence, by Theorem 3.7, its measure is no smaller than that of the former.

(It is easy to extend this method of proof to prove that a formula with $k$ clauses in which no letter appears both with and without a bar has this maximum measure if and only if each clause has one literal and no literals are repeated or, equivalently, no clauses are redundant.)

THEOREM 4.5. If $\Phi$ has the structure $\left\langle j_{1}, \cdots, j_{k}\right\rangle$, then

$$
m(\Phi) \geqq 2^{a-j_{1}}+2^{d-j_{2}-1}+\cdots+2^{d-j_{k}-(k-1)} .
$$

I prove first that Theorem 4.5 holds where $\Phi$ is interclausally consistent. The proof is by induction. If $\Phi$ is of structure $\left\langle j_{1}\right\rangle$, then equality holds. Assume, as an inductive hypothesis, that the measure of any formula $\Gamma$ of structure $\left\langle j_{1}, \cdots, j_{h-1}\right\rangle$ satisfies 


$$
m(\Gamma) \geq 2^{a-j_{1}}+2^{d-j_{2}-1}+\cdots+2^{d-j_{h-1}-(h-2)} .
$$

Where $\Phi_{h}$ is of structure $\left\langle j_{1}, \cdots, j_{h}\right\rangle$,

$$
m\left(\Phi_{h}\right)=m\left(\Phi_{h-1}\right)+m\left(\varphi_{h}\right)-m\left(\varphi_{h} \Phi_{h-1}\right) .
$$

The formula $\Phi_{h-1}$ has structure $\left\langle j_{1}, \cdots, j_{h-1}\right\rangle$ and so, by inductive hypothesis,

$$
m\left(\Phi_{h-1}\right) \geqq 2^{d-j_{1}}+2^{d-j_{2}-1}+\cdots+2^{d-j_{h-1}-(h-2)} .
$$

Also $m\left(\varphi_{h}\right)=2^{a-j_{h}}$. Therefore, it remains to prove that

$$
m\left(\varphi_{h} \Phi_{h-1}\right) \leqq 2^{a-j_{h}-1}+2^{a-j_{h}-2}+\cdots+2^{a-j_{h}-(h-1)} .
$$

The formula $\varphi_{h} \Phi_{h-1}$ is equivalent to $\varphi_{h} \Psi$, where $\Psi$ is obtained from $\Phi_{h-1}$ by deleting literals which appear in $\varphi_{h}$; we know that there must be at least one literal in each clause of $\varphi_{h-1}$ which does not occur in $\varphi_{h}$, for otherwise $\varphi_{h}$ would subsume another clause of $\Phi$ contrary to the assumption that $\Phi$ has structure $\left\langle j_{1}, \cdots, j_{h}\right\rangle$ and is therefore apparently irredundant. Therefore $\Psi$ has $h-1$ clauses and has no literals of $\varphi_{h}$. Since $\Phi$ is interclausally consistent, it has no letters both with and without bars; it follows that, since $\Psi$ has no literals of $\varphi_{h}$, it has no letters of $\varphi_{h}$. Thus,

$$
m\left(\varphi_{h} \Psi\right)=\frac{1}{2^{a}} m\left(\varphi_{h}\right) m(\Psi)=\frac{2^{a-j_{h}}}{2^{a}} m(\Psi)
$$

by Theorems 4.3 and 3.2. Since $\Psi$ has $h-1$ clauses and since no letter appears both with and without bars in $\Psi$, it follows from Theorem 4.4 that, regardless of the value of $h, m(\Psi) \leqq 2^{a-1}+2^{a-2}+\cdots+2^{a-(h-1)}$. Therefore,

$$
\begin{aligned}
m\left(\varphi_{h} \Phi_{h-1}\right) & =m\left(\varphi_{h} \Psi\right) \leqq \frac{2^{a-j_{h}}}{2^{a}}\left(2^{d-1}+2^{a-2}+\cdots+2^{a-(h-1)}\right. \\
& =2^{a-j_{h}-1}+2^{d-j_{h}-2}+\cdots+2^{d-j_{h}-(h-1)}
\end{aligned}
$$

which is what had to be proved.

To show that Theorem 4.5 still holds when $\Phi$ is not intercluasally consistent, I must discuss what happens to a formula when $d$ varies. I shall use the notation $m_{d}(\Phi)$ here (and only here) to denote the measure of $\Phi$ for a given $d$. From the definition of measure, it can be seen that $m_{a}(\Phi)=m_{d^{\prime}}(\Phi) \cdot 2^{a-d^{\prime}}$, assuming that $\Phi$ has at most min $\left(d, d^{\prime}\right)$ letters. Let $\Phi$ be a formula of structure $\left\langle j_{1}, \cdots, j_{k}\right\rangle$ which is not interclausally consistent. I have to prove that, for any $d$ not less than the number of distinct letters appearing in $\Phi$,

$$
m_{a}(\Phi) \geqq 2^{d-j_{1}}+2^{a-\jmath_{2}-1}+\cdots+2^{d-j_{k}-(k-1)} .
$$


Let $e$ be the number of letters appearing both with and without bars in $\Phi$; then $m_{a+e}(\Phi)=m_{a}(\Phi) 2^{c}$. There are at most $d+e$ distinct literals in $\Phi$, and so, by Theorem 4.2 , there is an interclausally consistent $\Phi^{\prime}$ of structure $\left\langle j_{1}, \cdots, j_{k}\right\rangle$ (with at most $d+e$ letters) such that $m_{d+e}\left(\Phi^{\prime}\right)$ $\leqq m_{a+e}(\Phi)$. But from what has been established it follows that

$$
m_{a+e}\left(\Phi^{\prime}\right) \geqq 2^{a+e-j_{1}}+2^{a+e-j_{2}-1}+\cdots+2^{a+e-j_{k}-(k-1)},
$$

which must now be true for $m_{d+e}(\Phi)$. Therefore,

$$
m_{a}(\Phi) \geqq 2^{a-j_{1}}+2^{a-j_{2}-1}+\cdots+2^{a-j_{k}-(k-1)} .
$$

This observation completes the proof of Theorem 4.5.

For $j_{k}+k \leqq d+1$, a formula with structure $\left\langle j_{1}, \cdots, j_{k}\right\rangle$ which has the minimum measure $2^{d-j_{1}}+2^{d-j_{2}-1}+\cdots+2^{d-j_{k}-(k-1)}$ has been exhibited in the literature, namely in Quine's paper [2]. Quine does not discuss the measure of formulas, but proves, in his Theorem 2, that his formula has the value truth in just the first $2^{a-j_{1}}+\cdots+2^{a-j_{k}-(k-1)}$ rows of the conventional truth table. By the well known connection between truth tables and developed normal formulas it follows that the measure of Quine's formula is this number. The construction of this formula which has no bars can be described as follows: the first clause has $j_{1}$ distinct letters, and, in general, the $h^{\text {th }}$ clause has all the letters of the $(h-1)^{\text {th }}$ clause except the last and enough letters which do not appear in any previous clause (at least one, since $j_{h-1} \leq j_{h}$ ) to make a total of $j_{h}$ distinct letters; the last letter of the $h^{\text {th }}$ clause is a letter which has not appeared previously. It follows that the last letter of any clause of Quine's formula appears in that clause only. For example, if $d=10$, the Quine formula of structure $\langle 1,1,3,3,6\rangle$ whose measure is $2^{9}+2^{8}+2^{5}+2^{4}+2^{0}$ is

$$
A_{1} \bigvee A_{2} \bigvee A_{3} A_{4} A_{5} \bigvee A_{3} A_{4} A_{6} \bigvee A_{3} A_{4} A_{7} A_{8} A_{9} A_{10}
$$

(It is possible to exploit the method used in proving Theorem 4.5 to prove that, for $j_{k}+k \leq d+1$, the only formulas with structure $\left\langle j_{1}, \cdots, j_{k}\right\rangle$ and measure $2^{a-j_{1}}+\cdots+2^{d-j_{k}-(k-1)}$ are those isomorphic to Quine's formula. The key property of Quine's formula is the fact that each clause $\varphi$ contains a literal, say $A_{q}$, such that $A_{q}$ is not in any other clause of the formula and all clauses followings $\varphi$ contain all the literals of $\varphi$ except $A_{q}$. In Quine's formula $A_{q}$ is the last letter of the clause. This property is necessary, as well as sufficient, in order to insure that the formula $\Psi$ in the proof of Theorem 4.5 has exactly $h-1$ clauses of one letter each, these letters being different from each other.)

One method of finding, for a given $d$ and for a given measure $m \leqq 2^{a}$, a simplest normal formula whose measure is $m$ is to construct some normal formula of measure $m$ and then calculate the measure of 
all simpler normal formulas. This method is impractical (although effective) unless there is some way of limiting the number of formulas whose measure must be calculated. A method of constructing a formula due to Quine was described two paragraphs above; from what Quine shows, it follows that for any given measure such a formula can be constructed. Although Quine's formula is not always a simplest formula with that measure, it can serve to start the search for such a formula. Then the bounds on the measure of formulas with given structures established in this section serve to limit the number of formulas among which the search is to be made, (although not enough to make this method practicable). Only formulas having some structure need be examined; normal formulas without any structure are not apparently irredundant and have shorter equivalent formulas or can be converted to a formula with structure by changing the order of disjuncts. Needless to say, once a formula has been examined, formulas isomorphic to it need not be. The following theorem will be of some help in the search, although not enough to make it practicable in all examples.

THEOREM 4.6. If a formula with some structure and with a least $h+1$ clauses, where $h+1 \leqq k$, has measure

$$
2^{d-j_{1}}+2^{d-j_{2}-1}+\cdots+2^{d-j_{k}-(k-1)}
$$

and if the first $h$ clauses of it have exactly $j_{1}, \cdots, j_{h}$, respectively, letters, then the $(h+1)^{\mathrm{st}}$ clause has at least $j_{h+1}$ letters.

Proof. A formula $\Phi$ of structure $\left\langle j_{1}, \cdots, j_{h}, j_{h+1}^{\prime}, j_{k+2}^{\prime}, \cdots\right\rangle$, where $j_{n+1}^{\prime}<j_{n+1}$, has a measure which satisfies, by Theorem 4.5 ,

$$
\begin{aligned}
m(\Phi) \geqq & 2^{a-j_{1}}+\cdots+2^{a-j_{h}-(h-1)}+2^{a-j^{\prime} h+1^{-h}}+\cdots \\
& >2^{a-j_{1}}+\cdots+2^{d-j_{h}-(h-1)}+2^{d-j_{h+1}-h}+\cdots+2^{d-j_{k}-(k-1)} .
\end{aligned}
$$

The last inequality is justified by the fact that the two expressions are each sums of powers of 2 with descending indices. As is well known about such expressions, since equality holds for the first $h$ terms, the inequality of the $(h+1)^{\text {st }}$ term is decisive.

5. Conjectures and a counterexample. The results of the previous sections lead to no practical method of findining a simplest formula with a given measure. But there are two conjectures which, if they are true, would be of some significance. Another conjecture which suggests itself rather naturally turns out to be false, as a counterexample of mine will show. (I must admit that these conjectures may turn on 
the definition of "simplicity" which has not be given precisely in this paper.)

A one-clause formula with $j$ literals, no two of which are of the same letter, has a measure $2^{a-j}$. Any formula with at least two noncontradictory clauses having this measure cannot have a non-contradictory clause with less than $j+1$ literals (by Theorem 4.1). Thus a oneclause formula is simpler than any formula with more clauses but with the same measure. My first conjecture, in its strong form, is that any normal formula $\Phi$ is simpler than any formula with the same measure but with more clauses. The weaker form is that $\Phi$ is at least a simple any such formula. I have no conterexample to either of these propositions, nor do I have any good reason to believe that either of them is true in general.

Let $r$ be the number of distinct letters of $\Phi$. Then $r \leqq d$, and $m(\Phi)$ is divisible by $2^{d-r}$. If. $2^{d-x}$ is the largest power of two which divides a given number $m$, then it is possible to find a formula with measure $m$ with just $x$ distinct letters. One example is Quine's formula with that measure (described near the end of $\S 4$ of this paper). But for some $\Phi, m(\Phi)$ is divisible by a power of two greater than $2^{a-r}$. For example, for $d \geqq 3, m\left(A_{1} A_{2} \bigvee A_{2} A_{3} \vee A_{1} A_{3}\right)=2^{a-1}$. In this example there is a simpler formula with the same measure, namely $A_{1}$. My second conjecture is that for any measure $m$, for any simplest formula $\Phi$ with measure $m, m$ is divisible by $2^{a-r}$. A weaker form of this conjecture is that, for any measure $m$, there exists a simplest formula $\Phi$ with measure $m$ such that $m$ is divisible by $2^{a-r}$.

A formula with two clauses which are each consistent but which contradict each other (because a letter appears with a bar in one and without a bar in the other) has a measure $2^{a}+2^{b}$, if there are $d-a$ and $a-b$ distinct literals in the respective clauses. If $a=b$ then a single clause formula with $d-a-1$ literals, no two of the same letter, has the same measure and is simpler. If $a>b$, then Quine's formula of measure $2^{a}+2^{b}$ has two clauses with $d-a$ and $d-b-1$, respectively, letters and is, therefore, simpler. A third conjecture that suggests itself is that, for any formula in which some letter appears both with and without a bar, there is another formula in which no letter appears both with and without a bar, which has the same measure and which is no less simple. However, for $d=6$ the formula

$$
A_{1} A_{2} \vee \bar{A}_{1} A_{3} A_{4} \bigvee A_{2} A_{5} A_{6}
$$

is simpler than any formula which has the same measure and which has no letter appearing both with and without a bar. (To verify this, the reader should note that Quine's formula with that measure has structure $\langle 2,2,3,3\rangle$. Using Theorems 4.6 and 4.1 the only possible 
structures for formulas which have the desired measure and are at least as simple as the displayed formula are $\langle 2,2,3\rangle,\langle 2,2,4\rangle$, $\langle 2,3,3\rangle$, and $\langle 2,2\rangle$. Since the desired measure is not divisible by two, and since $d=6$, there must be exactly six distinct letters in any formula with that measure: for there are at most six, since $d=6$; and there are at least six, by an observation made in this section. Therefore, the structure $\langle 2,2\rangle$ is excluded. Any formula with exactly six distinct letters in which no letter appears both with and without a bar is isomorphic to $A_{1} A_{2} \bigvee A_{1} A_{3} \bigvee A_{4} A_{5} A_{6}$ or $A_{1} A_{2} \bigvee A_{3} A_{4} \bigvee A_{1} A_{5} A_{6}$ if it has the structure $\langle 2,2,3\rangle$, is isomorphic to $A_{1} A_{2} \bigvee A_{3} A_{4} \bigvee A_{1} A_{3} A_{5} A_{6}$ or $A_{1} A_{2} \bigvee A_{1} A_{3} \bigvee A_{3} A_{4} A_{5} A_{6}$ or $A_{1} A_{2} \bigvee A_{1} A_{3} \bigvee A_{3} A_{4} A_{5} A_{6}$ if it has the structure $\langle 2,2,4\rangle$, and is isomorphic to $A_{1} A_{2} \bigvee A_{1} A_{3} A_{4} \bigvee A_{1} A_{5} A_{6}$ or $A_{1} A_{2} \bigvee A_{1} A_{3} A_{4}$ $\bigvee A_{2} A_{5} A_{6} \quad$ or $A_{1} A_{2} \bigvee A_{1} A_{3} A_{4} \bigvee A_{3} A_{5} A_{6}$ or $A_{1} A_{2} \bigvee A_{3} A_{4} A_{5} \bigvee A_{1} A_{3} A_{6}$ or $A_{1} A_{2}$ $\vee A_{3} A_{4} A_{5} \bigvee A_{3} A_{4} A_{6}$ if is has the structure $\langle 2,3,3\rangle$. But none of these formulas has the desired measure.)

\section{REFERENCES}

1. W. V. Quine, The problem of simplifying truth functions, Amer. Math. Monthly, 59 (1952), 521-531.

2. - Two theorems about truth functions, Bol. Soc. Mat. Mexicana, 10 (1953), $64-70$.

3. _ _ A way to simplify truth functions, Amer. Math. Monthly, 62 (1955), 627631.

4. R. K. Richards, Arithmetic operations in digital computere, New York, 1955. 


\section{PACIFIC JOURNAL OF MATHEMATICS}

EDITORS

H. L. ROYDEN

Stanford University

Stanford, California

R. A. Beaumont

University of Washington

Seattle 5 , Washington
A. R. Whiteman

University of Southern California

Los Angeles 7, California

E. G. Straus

University of California

Los Angeles 24, California

\section{ASSOCIATE EDITORS}
E. F. BECKENBACH
C. E. BURGESS
M. HALL
E. HEWITT
A. HORN
V. GANAPATHY IYER
R. D. JAMES
M. S. KNEBELMAN

L. NACHBIN

I. NIVEN

G. SZEKERES

T. G. OSTROM

M. M. SCHIFFER
F. WOLF

K. YOSIDA

\section{SUPPORTING INSTITUTIONS}

UNIVERSITY OF BRITISH COLUMBIA

CALIFORNIA INSTITUTE OF TECHNOLOGY

UNIVERSITY OF CALIFORNIA

MONTANA STATE UNIVERSITY

UNIVERSITY OF NEVADA

OREGON STATE COLLEGE

UNIVERSITY OF OREGON

UNIVERSITY OF SOUTHERN CALIFORNIA

\author{
STANFORD UNIVERSITY \\ UNIVERSITY OF UTAH \\ WASHINGTON STATE COLLEGE \\ UNIVERSITY OF WASHINGTON \\ AMERICAN MATHEMATICAL SOCIETY \\ CALIFORNIA RESEARCH CORPORATION \\ HUGHES AIRCRAFT COMPANY \\ THE RAMO-WOOLDRIDGE CORPORATION
}




\section{Pacific Journal of Mathematics}

\section{Vol. 7, No. $1 \quad$ January, 1957}

Richard Davis Anderson, Zero-dimensional compact groups of

homeomorphisms ................................... 797

Hans-Joachim Bremermann, Holomorphic functionals and complex

convexity in Banach spaces........................... 811

Hugh D. Brunk, G. M. Ewing and W. R. Utz, Minimizing integrals in

certain classes of monotone functions ................. 833

Philip David, Uniqueness theory for asymptotic expansions in general

regions ...................................... 849

Paul Erdős and Harold Nathaniel Shapiro, On the least primitive root of a

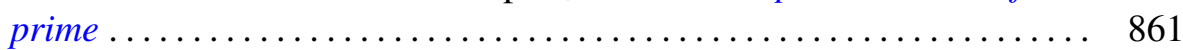

Watson Bryan Fulks, Regular regions for the heat equation ........... 867

William Robert Gaffey, A real inversion formula for a class of bilateral

Laplace transforms ................................ 879

Ronald Kay Getoor, On characteristic functions of Banach space valued random variables ................................. 885

Louis Guttman, Some inequalities between latent roots and minimax (maximin) elements of real matrices ...................... 897

Frank Harary, The number of dissimilar supergraphs of a linear graph .... 903

Edwin Hewitt and Herbert S. Zuckerman, Structure theory for a class of convolution algebras .................................. 913

Amnon Jakimovski, Some Tauberian theorems . . . . . . . . . . . . . . . . . 943

C. T. Rajagopal, Simplified proofs of "Some Tauberian theorems" of Jakimovski................................

Paul Joseph Kelly, A congruence theorem for trees ................. 961

Robert Forbes McNaughton, Jr., On the measure of normal formulas...... 969

Richard Scott Pierce, Distributivity in Boolean algebras .............. 983

Calvin R. Putnam, Continuous spectra and unitary equivalence ......... 993

Marvin Rosenblum, Perturbation of the continuous spectrum and unitary

equivalence................................... 997

V. N. Singh, Certain generalized hypergeometric identities of the

Rogers-Ramanujan type.......................

Peter Swerling, Families of transformations in the function spaces $H^{p} \ldots \ldots 1015$ 